\title{
La malattia ossea post-trapianto
}

\author{
M. Pasquali, A. Frasheri, S. Rotondi, L. Tartaglione, S. Mazzaferro
}

Dipartimento di Scienze Cardiovascolari, Respiratorie, Nefrologiche e Geriatriche, "Sapienza" Università di Roma, Roma

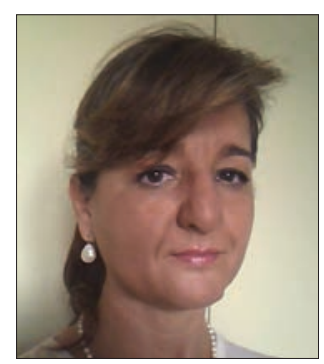

Marzia Pasquali

vanno aggiunti i fattori comuni alla popolazione generale, quali diabete, età e fumo.

\section{Persistenza di alterazioni del metabolismo minerale}

Dopo trapianto, il miglioramento del metabolismo minerale, in particolare a carico di calcemia, fosforemia, calcitriolo e PTH, è in genere rapido (2). Tuttavia a distanza di un anno, nonostante la normalizzazione della funzione renale, una percentuale di pazienti stimata tra il 15 e il $20 \%(3,4)$ presenta valori di PTH elevati, non di rado associati a ipercalcemia e ipofosforemia (5). La persistenza di iperparatiroidismo viene riferita allo sviluppo, durante l'uremia, di un tipo particolare di iperplasia ghiandolare, quella nodulare, che risulta resistente alla normalizzazione bioumorale secondaria al trapianto $(4,6)$. Infatti questo tipo di tessuto presenta una spiccata riduzione della espressione dei recettori del $\mathrm{Ca}$ e della vitamina $\mathrm{D}$, il che lo rende poco responsivo alla inibizione prodotta dai livelli normalizzati di calcio e calcitriolo (7). Inoltre, se dopo trapianto la funzione renale non è normale, possono permanere attivi quei meccanismi di stimolo dell'iperparatiroidismo che si osservano nella insufficienza renale cronica, quali riduzione del calcitriolo ed iperfosforemia. Non si deve poi trascurare la pos- sibilità di carenza di vitamina $\mathrm{D}$, dato che la popolazione trapiantata presenta frequentemente livelli ridotti di 25-idrossivitamina D (8).

Una complicanza frequente e particolare del post-trapianto, a volte presente anche dopo molti anni, è l'ipofosforemia, secondaria ad aumentata escrezione renale $(9,10)$. A volte anche livelli nell'ambito della norma nascondono un'aumentata fosfaturia, che potrebbbe riflettere un'aumentata mobilizzazione del fosforo dall'osso per garantirne i livelli ematici (11). Lipofosfatemia cronica e severa si associa ad aumento della apoptosi degli osteoblasti, a diminuita attività osteoblastica e a riduzione dell'osteoblastogenesi, tutti fattori che possono promuovere e mantenere le lesioni ossee post-trapianto (12). La patogenesi della ipofosforemia nel trapianto è complessa e già da alcuni anni si è riconosciuto che non dipende solamente dal persistere di iperparatiroidismo (13). L'FGF23 (fibroblast growth factor 23), una fosfatonina sintetizzata dall'osso e in grado di aumentare la fosfaturia e ridurre la sintesi di calcitriolo (14), viene recentemente proposta come possible causa di ipofosforemia nel trapianto. Infatti questa fosfatonina aumenta nei pazienti con insufficienza renale cronica (15) proporzionalmente al danno renale e il trapianto ne migliora i livelli, ma senza riuscire a normalizzarli (16). Altri possibili fattori coinvolti nella ipofosforemia del trapianto sono la denervazione renale (17) e la terapia immunosoppressiva (18).

\section{Farmaci immunosoppressori}

Glucocorticoidi. I glucocorticoidi determinano effetti negativi sull'osso mediante meccanismi diretti e indiretti. Con meccanismo diretto promuovono l'apoptosi degli osteoblasti e degli osteociti con conseguente riduzione del turnover e della sintesi ossea (19) e stimolano l'osteoclastogenesi tramite un aumento dei livelli del RANKL e una diminuizione del recettore decoy di RANKL, l'osteo- 
protegerina (20). Con meccanismo indiretto determinano una riduzione della funzione degli osteoblasti interferendo con la sintesi di osteocalcina, di collagene di tipo I e di fattori osteoanabolici quali l'IGF1 (21). Inoltre contribuiscono alla perdita ossea inducendo ipogonadismo ipogonadotropo (22) e negativizzando il bilancio del calcio (riduzione dell'assorbimento intestinale e aumentata escrezione renale) (23). L'uso degli steroidi si associa a una rapida perdita di osso durante i primi mesi dopo il trapianto renale, con maggiori effetti a livello dell'osso trabecolare (24).

Inibitori della calcineurina. Esperimenti in vivo su animali trattati con ciclosporina hanno mostrato una relazione fra farmaco, aumento del turnover e perdita ossea (25). Nell'uomo il trattamento con ciclosporina sembra aumentare sia l'attività osteoblastica che quella osteoclastica (26). Il trattamento con Tacrolimus sembra indurre sia differenziazione osteoblastica che, in presenza di elevati livelli di PTH, osteoclastica (27). In modelli sperimentali il trattamento con Tacrolimus comporta una severa perdita di massa ossea e alterato turnover (28). Sebbene alcuni studi clinici dimostrino un link tra trattamento con inibitori della calcineurina e osteoporosi (29, 30 ), non sono state trovate correlazioni tra fratture e uso di questi farmaci (31).

Sirolimus/Everolimus. Il Sirolimus aumenta la sintesi di osteoprotegerina e inibisce la maturazione degli osteoclasti (32), e inoltre l'inibizione di mTOR aumenta l'apoptosi degli osteoclasti (33). L'Everolimus inibisce la formazione osteoclastica e la differenziazione osteoblastica nei topi e in cellule ossee della linea umana in vitro. Nei ratti ovariectomizzati riduce la perdita di osso tramite diminuzione del riassorbimento osteoclastico (34). Non ci sono tuttavia evidenze cliniche di effetti ossei di questi farmaci nell'uomo.

\section{L'Osteodistrofia renale nel trapianto}

Il paziente trapiantato presenta dunque possibili fattori di rischio di osteodistrofia renale (ODR) che, ricordiamolo, indica qualunque tipo di patologia ossea riscontrabile nei malati renali e che, come tale, è parte integrante della sindrome detta "chronic kidney disease-mineral bone disorders" (o CKD-MBD). Questa sindrome è stata proposta recentemente per sottolineare il legame tra le lesioni ossee e metaboliche dell'uremia e l'aumentata morbilità e mortalità dei pazienti renali, inclusi i trapiantati (35). Nella nuova sindrome, i classici quattro differenti tipi di ODR - iperparatiroidimo o osteite fibrosa, osteomalacia, osso adinamico e quadro misto (36) - potrebbero essere catalogati in modo differente qualora si affermasse la nuova classificazione basata su tre parametri ritenuti funzionalmente rilevanti, e cioè mineralizzazione, turnover e volume osseo (Tab. I).

Classificazione a parte, è noto che i differenti tipi di osteopatia mostrano prevalenze diverse nell'IRC stadio 3-5 e nell'emodialisi (dove prevale l'osteite fibrosa) rispetto alla dialisi peritoneale (dove prevale l'osso adinamico) (37). Nel trapianto i pochi dati disponibili indicano il prevalere di una condizione di basso turnover, con o senza osteomalacia (38). In uno studio italiano, effettuato in soggetti trapiantati con contenuto minerale osseo indicativo di grave osteoporosi (T Score $>-4$ ), la diagnosi istologica includeva tutti i tipi di ODR mentre la lesione puramente osteoporotica, quella teoricamente più attesa, era limitata al 25\%. Questi dati, oltre ad evidenziare il persistere della ODR, indicano il limitato valore diagnostico della densitometria nei trapiantati (39). Ovviamente gravità e tipo di lesione istologica sono molto influenzate dalla malattia sviluppata durante la fase dialitica e che poi non guarisce col trapianto. Comunque

TABELLA I - CONFRONTO TRA CLASSIFICAZIONE STANDARD DELLA OSTEODISTROFIA RENALE E NUOVI CRITERI PROPOSTI NELLE RECENTI LINEE GUIDA

\begin{tabular}{|c|c|c|c|c|}
\hline \multicolumn{2}{|l|}{ Definizione classica } & \multicolumn{3}{|c|}{ Nuova classificazione K/DIGO } \\
\hline & & $\mathbf{M}$ & $\mathbf{T}$ & $\mathbf{V}$ \\
\hline Osteite-fibrosa & $\begin{array}{l}\text { Accelerato processo di mineralizzazione e formazione ossea } \\
\text { (associato a iperparatiroidismo) }\end{array}$ & Normale & Aumentato & $\begin{array}{l}\text { Aumentato/ } \\
\text { Normale }\end{array}$ \\
\hline Osteomalacia & $\begin{array}{l}\text { Conseguenza clinica del ridotto processo di mineralizzazione } \\
\text { della matrice organica ossea (caratteristica del deficit di Vitamina D) }\end{array}$ & Anormale & Basso & Normale \\
\hline Malattia dell'osso adinamico & Ridotto processo di formazione ossea e osteoide normale & Normale & Basso & Basso \\
\hline Osteodistrofia mista & $\begin{array}{l}\text { Associazione delle lesioni ossee tipiche sia dell'osteite-fibrosa che dell'o- } \\
\text { steomalacia }\end{array}$ & Anormale & Aumentato & Normale \\
\hline
\end{tabular}

ODR = Osteodistrofia renale $\mathrm{M}=$ Mineralizzazione, $\mathrm{T}=$ Turnover, $\mathrm{V}=$ Volume 
sia, come nel dializzato, la presenza di malattia ossea va considerata un fattore di rischio di fratture e calcificazioni vascolari $(40,41)$.

\section{Aspetti clinici}

Alterazioni biochimiche a parte, vari studi nei trapiantati evidenziano una elevata incidenza e prevalenza non solamente di dolore osseo diffuso ma anche di fratture (42). Il test diagnostico più diffuso per lo studio della malattia ossea nel trapianto è la misura della densità minerale (BMD) mediante DXA, grazie alla quale è stato possibile documentare, in modo non invasivo, la rapida diminuzione del contenuto di calcio nell'osso nei primi mesi dopo trapianto, in relazione alla più consistente terapia corticosteroidea (43). Tuttavia una certa riduzione annuale del BMD è evidenziabile anche nel lungo termine (44) verosimilmente in rapporto al persistere della malattia ossea, con i meccanismi descritti in precedenza. Ma anche in questo caso il dato clinico più rilevante è la non trascurabile frequenza di fratture. Una analisi retrospettiva su 1527 pazienti ha mostrato una incidenza del $19,1 \%$ in un periodo di 6,5 anni, con il 6,4\% di tutti i pazienti affetti da fratture multiple. L'incidenza aumentava nel tempo passando dal $12 \%$ entro i primi 5 anni, al 23\% dopo 15 anni dal trapianto (45). Secondo Ball et al l'incidenza di fratture nei trapiantati sarebbe più alta rispetto ai dializzati (46).

Quindi in questi pazienti è particolarmente importante, dal punto di vista clinico, ricercare le fratture ed in particolare quelle vertebrali che, è bene ricordarlo, sono quasi sempre asintomatiche. Queste fratture possono essere evidenziate con varie tecniche radiologiche, quali la stima visiva su radiografia standard della colonna (da parte di un operatore esperto), la morfometria vertebrale semiautomatica su radiografia standard e la morfometria automatica con tecnica densitometrica (DXA). Quest'ultima, per il fatto di essere automatica ed associata a minima esposizione radiologica, potrebbe diventare la tecnica di scelta per il follow-up di questi pazienti. In proposito occorre sottolineare la scarsa corrispondenza diagnostica tra fratture vertebrali e riscontro di valori ridotti di BMD $(47,48)$. Infatti la BMD è una valutazione solamente quantitativa dell'osso esaminato, mentre sappiamo che è molto importante anche la qualità del tessuto e che quest'ultima è influenzata dalle alterazioni del trofismo osseo tipiche della ODR (49). Data la limitata accuratezza diagnostica, la DXA in questi pazienti può essere considerata solamente un utile strumento di follow-up, una volta che sia stata posta la diagnosi istologica.

\section{Terapia}

La cura della malattia ossea post-trapianto non può non tenere in debito conto i vari aspetti patogenetici ricordati, avendo sempre in mente che stiamo curando una forma di osteodistrofia e non una osteoporosi steroidoindotta (50).

Le maggiori evidenze disponibili riguardano il trattamento della rapida perdita minerale ossea che si osserva subito dopo il trapianto con schemi che prevedono l'uso della vitamina $\mathrm{D}$, associata o meno a supplementazione di calcio e bisfosfonati (51-53). La somministrazione del solo colecalciferolo (25.000 UI/mese) non sembra in grado di prevenire la perdita di massa ossea dopo il trapianto, mentre facilita la normalizzazione dei livelli di PTH (54). L'uso di metaboliti della vitamina D, come calcidiolo e calcitriolo, è risultato più efficace nel ridurre la perdita ossea, verosimilmente per l'antagonismo sugli effetti dei glucocorticoidi a livello intestinale e paratiroideo (51). L'uso del calcitriolo $(0.25 \mathrm{ug} / \mathrm{di})$ in aggiunta a supplementi di calcio $(1 \mathrm{~g} / \mathrm{di})$ ha dimostrato un effetto positivo sulla perdita di massa ossea a livello lombare e femorale, nei primi 6 mesi dopo il trapianto (52). L'utilizzo dei bisfosfonati nelle prime fasi post-trapianto, prevalentemente con preparati somministrati per via endovenosa, ha dato i risultati più significativi. Fan et al in 26 pazienti randomizzati a pamidronato $(0,5 \mathrm{mg} / \mathrm{kg}$ e.v $)$ somministrato al tempo 0 e a un mese dopo il trapianto, ovvero a placebo, hanno dimostrato una perdita di massa ossea sia a livello lombare che femorale significativamente inferiore nei pazienti trattati rispetto al gruppo di controllo (53). Coco et al hanno invece dimostrato l'efficacia del trattamento precoce con pamidronato associato a calcitriolo più calcio per os nel preservare la massa ossea a 6 e a 12 mesi. Tuttavia il trattamento con pamidronato si associa ad aumentata incidenza di osso adinamico e non sono disponibili dati sulla successiva incidenza di fratture (54). In effetti i pazienti trapiantati, come detto, hanno già una tendenza a sviluppare osso a basso turnover e di conseguenza l'uso dei bisfosfonati potrebbe essere controindicato. L'uso limitato ai primi mesi dopo trapianto potrebbe comunque trovare indicazione nei pazienti con bassa massa ossea o con aumentato riassorbimento al momento del trapianto (55).

Diverso è il tema della terapia della malattia ossea nel lungo termine dopo trapianto. In un trial randomizzato su 30 pazienti con età media del trapianto di 10 anni, il calcitriolo associato al calcio carbonato non ha comportato un significativo miglioramento della perdita ossea a livello del radio distale, lombare e femorale. Tuttavia è stata riscontrata una soppressione degli osteoclasti ed una sostanziale stabilità del volume trabecolare e dello 
spessore parietale dell'osso nel gruppo in terapia con calcitriolo (56). Non è tuttavia chiaro se tale terapia, nel lungo termine, influenza il rischio di fratture, mentre occorre considerare il rischio di ipercalcemia. Anche i bisfosfonati sono stati impiegati nei pazienti già trapiantati da tempo. L'etidronato per via orale ha comportato un aumento di densità ossea sia lombare che femorale in pazienti al secondo anno di trapianto (57). Analogamente efficace è risultato l'alendronato $(58,59)$. Un simile incremento della massa ossea, sia a livello lombare che femorale, è stato dimostrato sia con calcitriolo che con alendronato in pazienti trapiantati da circa 8-9 anni (60). Rispetto ai bisfosfonati occorre ricordare che la loro clearance è esclusivamente renale e che pertanto ne è controindicato l'uso in caso di insufficienza renale. Per il potenziale effetto peggiorativo sulle forme di osteopatia adinamica, le linee guida più recenti ne consigliano l'uso solo previa accertamento istologico (37).

Anche la terapia dell'iperparatiroidismo persistente dopo trapianto presenta alcune peculiarità. Essendo in genere associato a ipercalcemia, è problematico l'uso dei metaboliti attivi della vitamina $\mathrm{D}$. Teoricamente potrebbe essere preferibile usare gli analoghi non calcemizzanti, ma al momento non vi sono studi disponibili sulla efficacia e tollerabilità. Più favorevole si presenta l'uso dei calciomimetici che oltre a ridurre l'ipercalcemia aumentano i livelli di fosforo (il che potrebbe essere desiderabile vista la non rara presenza di ipofosforemia). Tuttavia nelle segnalazioni presenti in letteratura il calo di PTH sembra essere transitorio, legato alla costante somministrazione del farmaco e associato ad aumento della calciuria $(61,62)$.

In conclusione, nel primo anno dopo trapianto sembra ragionevole l'uso di supplementi di calcio, vitamina D e bisfosfonati (salvo presenza di insufficienza renale), specie nei pazienti che si presentano con bassi valori di densità minerale ossea. Più complicato l'uso generalizzato nel lungo termine, in particolare dei bisfosfonati. Per questi ultimi, oltre ad evitare l'uso in caso di insufficienza renale, è raccomandabile eseguire controlli istologici prima e dopo la terapia. Una precoce riduzione o sospensione del trattamento con corticosteroidi è comunque desiderabile specie nei casi con bassa densità minerale ossea.

\section{Indirzzo degli Autori:}

Sandro Mazzaferro, MD

Dipartimento di Scienze Cardiovascolari,

Respiratorie Nefrologiche e Geriatriche

"Sapienza" Università di Roma

Viale del Policlinico 155

Roma

sandro.mazzaferro@uniroma1.it

\section{Bibliografia}

1. Monier-Faugere MC, Mawad H, Qi Q, et al. High prevalence of low bone turnover and occurrence of osteomalacia after kidney transplantation; J Am Soc Nephrol 2000; 11: 1093-9.

2. Messa P, Cafforio C, Alfieri C. Impatto clinico dell'ipercalcemia nel trapianto renale. G Ital Nefrol 2010; 27: 47-55.

3. Evenepoel P, Claes K, Kuypers D, et al. Natural history of parathyroid function and calcium metabolism after kidney transplantation: a single-centre study. Nephrol Dial Transplant 2004; 19: 1281-7.

4. Messa P, Sindici C, Cannella G, et al. Persistent secondary hyperparathyroidism after renal transplantation. Kidney Int 1998; 54: 1704-13.

5. Evenepoel P, Van Den Bergh B, Naesens M, et al. Calcium metabolism in the early posttransplantation period. Clin J Am Soc Nephrol 2009; 4: 665-72.

6. McCarron DA, Muther RS, Lenfesty B, Bennett WM. Parathyroid function in persistent hyperparathyroidism: relationship to gland size. Kidney Int 1982; 22: 662-70.

7. Gogusev J, Duchambon P, Hory B, et al. Depressed expression of calcium receptor in parathyroid gland tissue of patients with hyperparathyroidism. Kidney Int 1997; 51: 328-36.

8. Stavroulopoulos A, Cassidy MJ, Porter CJ, et al. Vitamin D status in renal transplant recipient. Am J Transpl 2007; 7: 2546-52.

9. Felsenfeld AJ, Gutman RA, Drezner M, Llach F. Hypophosphatemia in long-term renal transplant recipients: effects on bone histology and 1, 25-dihydroxycholecalciferol. Miner Electrolyte Metab 1986; 12: 333-341.

10. Levi M. Post-transplant hypophosphatemia. Kidney Int 2001; 59: 2377-87.

11. Takeda E, Taketani Y, Sawada N, et al. The regulation and function of phosphate in the human body. Biofactors 2004; 21: 345-55.

12. Kunzendorf U, Krämer BK, Arns W, et al. Bone disease after renal transplantation. Nephrol Dial Transplant 2008; 23: 450-8.

13. Green J, Debby H, Lederer E, et al. Evidence for a PTHindependent humoral mechanism in post-transplant hypophosphatemia and phosphaturia. Kidney Int 2001; 60: 1182-96.

14. Shimada T, Urakawa I, Yamazaki Y, et al. FGF-23 transgenic mice demonstrate hypophosphatemic rickets with reduced expression of sodium phosphate cotransporter type IIaq. Biochem Biophys Res Commun 2004; 314: 40914.

15. Imanishi $\mathrm{Y}$, Inaba $\mathrm{M}$, Nakatsuka $\mathrm{K}$, et al. FGF-23 in patients with endstage renal disease on hemodialysis. Kidney Int 2004; 65: 1943-6. 
16. Bhan I, Shah A, Holmes J, et al. Post-transplant hypophosphatemia: tertiary 'hyper-phosphatoninism'? Kidney Int 2006; 70:1486-94.

17. Mann KJ, Rybczynska A, Berndt TJ, et al. Renal denervation enhances the phosphaturic effect of parathyroid hormone. Miner Electrolyte Metab 1991;17: 16-20.

18. Graf H, Kovarik J, Stummvoll HK, et al. Handling of phosphate by the transplanted kidney. Proc Eur Dial Transplant Assoc 1979; 16: 624-9.

19. Weinstein RS, Jilka RL, Parfitt AM, Manolagas SC. Inhibition of osteoblastogenesis and promotion of apoptosis of osteoblast and osteocytes by glucocorticoids. Potential mechanisms of their deleterious effects on bone. J Clin Invest 1998; 102: 274-82.

20. Canalis E. Mechanism of glucocorticoid action in bone: implications to glucocorticoid-induced osteoporosis. J Clin Endocrinol Metab 1996; 81: 3441-7.

21. Delany AM, Durant D, Canalis E. Glucocorticoid suppression of IGF1 transcription in osteoblast. Mol Endocrinol 2001; 15: 1781-9.

22. Sakakura M, Takebe K, Nakagawa S. Inhibition of luteinizing hormone secretion induced by synthetic LRH by long-term treatment with glucocorticoid in human subjects. J Clin Endocrinol Metab 1975; 40: 774-9.

23. Suzuki Y, Ichikawa Y, Saito E, Homma M. Importance of increased urinary calcium excretion in the development of secondary hyperparathyroidism of patients under glucocorticoid therapy, Metabolism 1983; 32: 151-6.

24. Julian BA, Laskow DA, Dubovsky J, et al. Rapid loss of vertebral mineral density after renal transplantation. $\mathrm{N}$ Engl J Med 1991; 325: 544-50.

25. Movsowitz C, Epstein S, Fallon M, et al. Cyclosporin-A in vivo produces severe osteopenia in the rat: effect of dose and duration of administration. Endocrinology 1988; 123: 2571-7.

26. Aubia J. Bone histology in renal transplamt patients receiving cyclosporin. Lancet 1988; 1: 1048-9.

27. Tang L, Ebara S, Kawasaki S, et al. FK506 enhanced osteoblastic differentiation in mesenchymal cells. Cell Biol Int 2002; 26: 75-84.

28. Kirino S, Fukunaga J, Ikegami S, et al. Regulation of bone metabolism in immunosoppressant (FK506)-treated rats. J Bone Miner Metab 2004; 22: 554-60.

29. Ugur A, Guvener N, Iهiklar I, et al. Osteoporosis after renal transplantation single center experience, Transplantation. 2001; 71: 645-9.

30. Marcén R, Caballero C, Pascual J, et al. Lumbar mineral density in renal transplant patients on neoral and tacrolimus: a four year prospective study. Transplantation 2006; 81: 826-31.

31. Patel S, Kwan JT, McCloskey E, et al. Prevalence and causes of low bone density and fractures in kidney transplant patients. J Bone Miner Res 2001; 16:1863-70.

32. Hofbauer LC, Shui C, Riggs BL, et al. Effects of immunosoppressant on receptor activator of NF-kappaB ligand and osteoprotegerin production by human osteoblastic and coronary artery smooth muscle cells. Biochem Biophys Res Commun 2001; 280: 334-9.

33. Glantschnig H, Fisher JE, Wesolowski G, et al. TNFalpha and RANK ligand promotes osteoclast survival by signalling throught mTOR/S6 kinase. Cell Death Differ 2003; 10: 1165-77.

34. Kneissel M, Luong-Nguyen NH, Baptist M, et al. Everolimus supresses cancellous bone loss, bone resorption and cathepsin $\mathrm{K}$ expression by osteoclast. Bone 2004; 35:1144-56.

35. Moe S, Drüeke T, Cunningham J, et al. Definition, evaluation, and classification of renal osteodystrophy: a position statement from Kidney Disease: Improving Global Outcomes (KDIGO). Kidney Int 2006; 69: 1945-53.

36. Hruska KA, Teitelbaum SL. Renal osteodistrophy. N Engl J Med 1995; 333: 166-74.

37. KDIGO clinical practice guideline for the diagnosis, evaluation, prevention, and treatment of chronic kidney disease -mineral and bone disorder (CKD-MBD). Kidney Int 2009; 76 (Suppl 113): S3-S130.

38. Monier-Faugere M C, Mawad H, Qi Q, et al. High prevalence of low bone turnover and occurrence of osteomalacia after kidney transplantation. J. Am. Soc. Nephrol 2000; 11: 1093-9.

39. Rolla D, Ballanti P, Marsano L, et al. Bone disease in longterm renal transplant recipients with severe osteopenia: a cross-sectional study. Transplantation 2006; 81: 915-21.

40. London GM, Marty C, Marchais SJ, Guerin AP, Metivier F, de Vernejoul MC. Arterial calcifications and bone histomorphometry in end-stage renal disease.J Am Soc Nephrol 2004; 15(7): 1943-51.

41. Adragao T, Herberth J, Monier-Faugere MC, Branscum AJ, Ferreira A, Frazao JM, Dias Curto J, Malluche HH. Low bone volume a risk factor for coronary calcifications in hemodialysis patients Clin J Am Soc Nephrol 2009; 4(2): 254-7

42. Malluche HH, Monier-Faugere MC, Herberth J. Bone disease after renal transplantation. Nat Rev Nephrol 2010; 6(1): 32-40.

43. Mikuls TR, Julian BA, Bartolucci A, Saag KG. Bone mineral density changes within six months of renal transplantation. Trasplantation 2003; 75: 49-54.

44. Pichette V, Bonnardeaux A, Prudhomme L, et al. Longterm bone loss in kidney transplant recipients: a crosssectional and longitudinal study. Am J Kidney Dis 1996; 28: 105-14.

45. O’Shaughnessy EA, Dahl DC, Smith CL, Kasiske BL. Risk factors for fractures in kidney transplantation. Tran- 
splantation 2002; 74: 362-6.

46. Ball AM, Gillen DL, Sherrard D, et al. Risk of hip fracture among dialysis and renal transplant recipients. JAMA 2002; 288: 3014-8.

47. Mazzaferro S, Diacinti D, Proietti E, et al. Morphometric X-ray absorptiometry in the assessment of vertebral fractures in renal transplant patients. Nephrol Dial Transplant 2006; 21: 466-71.

48. Grotz WH, Mundinger FA, Gugel B, et al. Bone fracture and osteodensitometry with dual X-ray absorptiometry in kidney transplant recipients. Transplantation 1994; 58: 912-5.

49. Nawrot-Wawrzyaniak K, Varga F, Nader A, et al. Effects of Tumor-Induced Osteomalacia on the Bone Mineralization Process. Calcif Tissue Int 2009; 84: 313-23.

50. Weisinger J, Carlini R, Rojas E, Bellorin-Font E. Bone disease after renal transplantation. Clin J Am Soc Nephrol 2006; 1: 1300-13.

51. Talalaj M, Gradowska L, Marcinovska E, et al. Efficiency of preventive treatment of glucocorticoid-induced osteoporosis with 25-hydroxyvitamin $\mathrm{D}_{3}$ and calcium in kidney transplant patients. Transplant Proc 1996; 28: 3485-7.

52. De Seveaux RG, Hoitsma AJ, Corstens FH, Wetzels JF. Treatment with vitamin $\mathrm{D}$ and calcium reduces bone loss after renal transplantation. J Am Soc Nephrol 2002; 13: 1608-14.

53. Torres A, Garcia S, Gomez A, et al. Treatment with intermittent calcitriol and calcium reduces bone loss after renal transplantation. Kidney Int 2004; 65: 705-12.

54. Wissing KM, Broeders N, Moreno-Reyes R, et al. A controlled study of vitamin $\mathrm{D}_{3}$ to prevent bone loss in renal-transplant patients receiving low doses of steroids. Transplantation 2005; 79: 108-15.

55. Fan SL, Almond MK, Ball E, et al. Pamidronate therapy as prevention of bone loss following renal transplantation.
Kidney Int 2000; 57: 684-90.

56. Coco M, Glicklich D, Faugere MC, et al. Prevention of bone loss in renal transplant recipients. A prospective, randomized trial. J Am Soc Nephrol 2003; 14: 2669-76.

57. Schwarz C, Mitterbauer C, Heinze G, et al. Nonsustained effect of short-term bisphosphonate therapy on bone turnover three years after renal transplantation. Kidney Int 2004; 65: 304-9.

58. Cueto-Manzano AM, Konel S, Freemont AJ, et al. Effect of 1,25-dihydroxyvitamin D3 and calcium carbonate on bone loss associated with long-term renal transplantation. Am J Kidney Dis 2000; 35: 227-36.

59. Arlen DJ, Lambert K, Ioannidis G, Adachi JD. Treatment of established bone loss after renal transplantation with etidronate.Transplantation 2001; 71: 669-73.

60. Koc M, Tuglular S, Arikan H, et al. Alendronate increases bone mineral density in long-term renal transplant recipients. Transplant Proc 2002; 34: 211-3.

61. Giannini S, D'Angelo A, Carraro G, et al. Alendronate prevents further bone loss in renal transplant recipients. J Bone Mineral Res 2001; 16: 211-7.

62. Jeffery JR, Leslie WD, Karpinski ME, Nickerson PW, Rush DN. Prevalence and treatment of decreased bone density in renal transplant recipients: a randomized prospective trial of calcitriol versus alendronate. Transplantation 2003; 7: 1498-02.

63. Kruse AE, Eisenberg U, Frey FJ, Mohaupt MG. The calcimimetic cinacalcet normalizes serum calcium in renal transplant patients with persistent hyperparathyroidism. Nephrol Dial Transplant 2005; 20: 1311-4.

64. Serra AL, Schwarz AA, Wick FH, et al. Successful treatment of hypercalcemia with cinecalcet in renal transplantation with persistent hyperparathyroidism. Nephrol Dial Transplantat 2005; 20; 1315-9. 